flexible-dose study, dasotraline demonstrated significant efficacy in patients with BED. We now report an analysis from this study of the effect of dasotraline on binge-related obsessions and compulsions.

METHOD: Patients with moderate-to-severe BED, based on DSM-5 criteria, were randomized to 12 weeks of double-blind, placebo controlled, treatment with flexible doses of dasotraline $(4,6$, and $8 \mathrm{mg} / \mathrm{d})$. The primary efficacy measure was number of binge-eating days/ week; secondary measures included the Binge Eating Clinical Global Impression of Severity (BE-CGI-S) score and the Yale-Brown Obsessive-Compulsive Scale Modified for Binge-Eating (Y-BOCS-BE), a validated, 10-item interviewer-administered measure designed to assess the severity of obsessional thoughts and compulsive behaviors related to binge eating. Change from baseline in efficacy measures in the Intent-to-treat (ITT) population were analyzed using a mixed model for repeated measures (MMRM) analysis.

RESULTS: The ITT population consisted of 317 patients (female, 84\%; mean age, 38.2 years). LS mean reduction from baseline in number of Binge Eating (BE) days per week was significantly greater for dasotraline vs. placebo at week 12 (-3.74 vs. $-2.75 ; \mathrm{P}<0.0001$; effect size $[\mathrm{ES}]=0.74$; primary endpoint); week 12 change was significantly greater for dasotraline vs. placebo on the Y-BOCS-BE total score (-17.05 vs. -9.88; $\mathrm{P}<0.0001$; ES, 0.96), the obsession subscale score (-8.32 vs. -4.58 ; $\mathrm{P}<0.0001 ; \mathrm{ES}, 0.95)$, and the compulsion subscale score (-8.69 vs. -5.35; $\mathrm{P}<0.0001 ; \mathrm{ES}, 0.87)$. All 10 YBOCS-BE items were significantly improved on dasotraline vs. placebo at week $12(\mathrm{P}<0.001$ for all comparisons; with effect sizes ranging from 0.54 to 0.90). At Week 12 (LOCF), for dasotraline and placebo, $52.3 \%$ and $18.4 \%$ of patients, respectively, had a BE-CGI-S score of 1 ("normal; not at all ill"; NNT=3). At endpoint, for patients with a global illness severity score of 1 , the corresponding mean Y-BOCS-BE total scores were 0.5 and 0.7 for dasotraline and placebo, respectively, indicating that when BED illness severity approaches "normal, not at all ill", binge-related obsessions and compulsions demonstrate comparably low levels of severity.

CONCLUSION: In this placebo-controlled, 12-week study of patients with moderate-to-severe binge eating disorder, treatment with dasotraline $(4-8 \mathrm{mg} / \mathrm{d})$ was associated with significant and clinically meaningful reduction in binge-related obsessional thoughts and compulsive behaviors.

Clinicaltrials.gov number: NCT02564588

Funding Acknowledgements: Supported by funding from Sunovion Pharmaceuticals Inc.
170

\section{Efficacy and Safety of Dasotraline in Adults with Binge-Eating Disorder: A Randomized, Double-blind, Fixed-dose Trial}

Joyce Tsai, PhD'; Brad Navia, $M D, P h D^{\prime}$; Susan L McElroy, $\mathrm{MD}^{2}$; James I Hudson, $M D^{3}$; Carlos M. Grilo, $\mathrm{PhD}^{4}$; Robert Goldman, $\mathrm{PhD}^{\prime}$; Ling Deng, PhD ${ }^{\prime}$; Justine Kent, $M D^{\prime}$; and Antony Loebel, $M D^{\prime}$

${ }^{1}$ Sunovion Pharmaceuticals Inc., Marlborough, MA

${ }^{2}$ Lindner Center of HOPE, Mason, $\mathrm{OH}$; and University of Cincinnati College of Medicine, Cincinnati, $\mathrm{OH}$

${ }^{3}$ McLean Hospital \& Harvard Medical School, Belmont, MA

${ }^{4}$ Department of Psychiatry, Yale University School of Medicine, New Haven, CT

ABSTRACT: Background: Dasotraline is a long-acting dopamine/norepinephrine reuptake inhibitor with a PK profile characterized by slow absorption and a $\mathrm{t}^{1 / 2}$ of 47-77 hours, permitting once-daily dosing. In a previous flexible dose study, dasotraline demonstrated significant efficacy in the treatment of binge-eating disorder (BED). The aim of this confirmatory fixed-dose study was to evaluate efficacy and safety of dasotraline in the treatment of patients with BED.

METHODS: Patients meeting DSM-5 criteria for BED were randomized to 12 weeks of double-blind treatment with dasotraline $(4 \mathrm{mg} / \mathrm{d}$ or $6 \mathrm{mg} / \mathrm{d}$ ), or placebo. The primary efficacy endpoint was change in number of binge-eating days per week at week 12 . Secondary efficacy endpoints included changes at Week 12 on the Binge Eating Clinical Global Impression of Severity Scale (BE-CGI-S), the YaleBrown Obsessive-Compulsive Scale Modified for Binge Eating (Y-BOCS-BE), and the proportion of patients with $100 \%$ cessation of binge-eating episodes during the final 4 weeks of treatment. Efficacy was assessed using an MMRM analysis (and a logistic regression model for cessation) with a pre-specified sequential testing procedure used to control overall type I error rate.

RESULTS: A total of 486 were in the ITT population (dasotraline $6 \mathrm{mg} / \mathrm{d}(\mathrm{N}=162), 4 \mathrm{mg} / \mathrm{d}(\mathrm{N}=161)$, or placebo $(\mathrm{N}=163)$. At week 12, treatment with dasotraline was associated with significant reduction in number of bingeeating days per week in the $6 \mathrm{mg} / \mathrm{d}$ group vs. placebo (-3.5 vs. $-2.9 ; \mathrm{P}=0.0045)$, but non-significant improvement in the $4 \mathrm{mg} / \mathrm{d}$ group vs. placebo $(-3.2 ; \mathrm{P}=0.12)$. Greater improvement was observed vs. placebo for dasotraline 6 $\mathrm{mg} / \mathrm{d}$ and $4 \mathrm{mg} / \mathrm{d}$, respectively, on the BE-CGI-S $(\mathrm{P}<0.01$ and $\mathrm{P}<0.03)$ and the $\mathrm{Y}-\mathrm{BOC}-\mathrm{BE}(\mathrm{P}<0.001$ and $\mathrm{P}<0.02$; all $\mathrm{P}$-values were nominal, not adjusted for multiplicity). The proportion of patients who achieved 4-week cessation of binge-eating episodes was only significant for the 
dasotraline $6 \mathrm{mg}$ in the completer population $(\mathrm{P}<0.05$; post-hoc analysis) but was not significant for either dose of dasotraline vs. placebo when drop-outs were included in the analysis. The most common adverse events on dasotraline $6 \mathrm{mg} / \mathrm{d}$ and $4 \mathrm{mg} / \mathrm{d}$ were combined insomnia (early, middle, late), dry mouth, headache, decreased appetite, nausea, and anxiety. Changes in systolic and diastolic blood pressure were minimal. Mean baseline to endpoint changes in supine pulse rate on dasotraline $6 \mathrm{mg} / \mathrm{d}$ and $4 \mathrm{mg} / \mathrm{d}$ vs. placebo was $+6.2 \mathrm{bpm}$ and +4.8 vs. $+0.2 \mathrm{bpm}$.

CONCLUSIONS: In this 12-week, placebo-controlled, fixeddose study, treatment with dasotraline $6 \mathrm{mg} / \mathrm{d}$ was associated with a significant reduction in frequency of bingeeating days per week; efficacy was not demonstrated for the $4 \mathrm{mg}$ dose. Treatment with both doses of dasotraline resulted in improvement in the Y-BOCS-BE and the BE-CGI-S. Dasotraline was safe and generally welltolerated at both doses; most common adverse events were insomnia, dry mouth and headache.

Clinicaltrials.gov: NCT03107026

Funding Acknowledgements: Supported by funding from Sunovion Pharmaceuticals Inc.

\section{1}

\section{Buprenorphine - A Treatment for Psychic Pain and Suicidal Ideation?}

Ori-Michael Benhamou, $M D^{\prime}$; Sean Lynch, $B A^{\prime}$; and Lidia Klepacz, $M D^{\prime}$

${ }^{1}$ Psychiatry Department, Westchester Medical Center, New York Medical College, Valhalla NY

ABSTRACT: Buprenorphine (BPN) is an opiate medication that is increasingly used in the management of Opioid Use Disorder and pain disorders. This case report highlights the acute efficacy of using buprenorphine-naloxone (BPN-NAL) to reverse anhedonia and suicidal ideation in an individual with OUD, chronic pain and severe suicide attempts.

We present a case of a 39-year-old male with a history of bipolar disorder, several lethal suicide attempts and polysubstance abuse, who presented to the hospital after self-immolation, burning $45 \%$ total body surface area. $\mathrm{He}$ was admitted to the burn unit for three months, reporting continual anhedonia, suicidal ideation, and flashbacks of seeing and feeling himself on fire. He also endorsed chronic pain and hopelessness.

Upon transfer to the behavioral health unit, his symptoms persisted, despite trials of quetiapine, mirtazapine, methadone, oxycodone and prazosin. In consultation with pain management, he was initiated on sublingual BPN-NAL 8mg-2mg treatment as a transition from methadone; he immediately reported improvement in depressive symptoms and a reduction in pain. He was titrated on BPN-NAL and continued to report diminished pain and resolution of depression. Furthermore, his irritability was lessened and he newly cooperated with staff, participating in unit activities. Upon discharge, he exhibited stable mood, adequate pain control and the elimination of suicidal thoughts as well as a proactive drive for substance abuse treatment.

This case describes the significance of BPN on relieving psychic pain and stabilizing mood in a chronically suicidal patient. We speculate that BPN's pharmacokinetic properties terminate the cycle of short-term opioid-induced analgesia and euphoria with opioid withdrawal-induced hyperalgesia and dysphoria. This results in a steady treatment of pain, as well as maintaining the dopaminergic system, symptomatically translating to mood stabilization and annulling suicidal ideation.

172

\section{A Phase 3, Multicenter Study to Assess the Long-Term Safety, Tolerability, and Efficacy of Olanzapine/Samidorphan in Patients with Schizophrenia}

Sergey Yagoda, $\mathrm{PhD}^{\prime}$; Christine Graham, $\mathrm{PhD}{ }^{\prime}$; Adam Simmons, $\mathrm{MPH}^{2}$; Christina Arevalo, $\mathrm{MBA}^{3}$; Yansong Cheng, $\mathrm{PhD}^{4}$; and David McDonnell, $\mathrm{MD}^{5}$

${ }^{1}$ Associate Medical Director, Clinical Research, Alkermes, Inc.

${ }^{2}$ Director, Clinical Program Management, Clinical

Operations, Alkermes, Inc.

${ }^{3}$ Senior Clinical Trial Manager, Clinical Operations, Alkermes, Inc.

${ }^{4}$ Associate Director, Biostatistics, Alkermes, Inc.

${ }^{5}$ Executive Medical Director, Clinical Science, Alkermes Pharma Ireland Limited

ABSTRACT: Background: ALKS 3831, a combination of olanzapine and samidorphan (OLZ/SAM), is in development for the treatment of schizophrenia and is intended to provide the antipsychotic efficacy of olanzapine while mitigating olanzapine-associated weight gain. We report the safety, tolerability, and efficacy of OLZ/SAM in patients with schizophrenia in a phase 3, 52-week, open-label extension study.

METHODS: Patients aged 18-70 years who completed a previous phase 3, 4-week, inpatient acute efficacy study were switched from OLZ/SAM, olanzapine, or placebo to OLZ/SAM. Study assessments included adverse events (AEs), weight, clinical laboratory testing, and Positive and Negative Syndrome Scale (PANSS) and Clinical Global Impression-Severity (CGI-S) scores. 